\title{
Discrepant Findings of Clozapine Effects on Prepulse Inhibition of Startle: Is It the Route or the Rat?
}

Neal R. Swerdlow, M.D., Ph.D., Geoffrey B. Varty, Ph.D., and Mark A. Geyer, Ph.D.

Studies examined methodological differences that might account for discrepant reports related to the ability of clozapine to restore prepulse inhibition (PPI) of acoustic startle in apomorphine (APO)-treated rats. Changes in PPI after APO and clozapine were compared in SpragueDawley (SD) versus Wistar rats. In SD rats, intraperitoneal administration of clozapine (4-12 $\mathrm{mg} / \mathrm{kg}$ ) completely reversed the PPI-disruptive effects of APO $(0.5$ $\mathrm{mg} / \mathrm{kg}$ ), with significant effects evident at the lowest dose of clozapine. Compared to SD rats, Wistars exhibited a relatively weaker (but statistically significant) disruption of
PPI with the same or higher doses of APO and were also less sensitive to the PPI-restorative effects of clozapine. Clozapine administered via subcutaneous route completely restored PPI after APO treatment in SD rats. Discrepant findings with this model can be attributed to differences in rat strain; SD rats exhibit patterns of drug responses in this model that are optimal for examining profiles of putative atypical antipsychotics. [Neuropsychopharmacology 18:50-56, 1998] (c) 1998 American College of Neuropsychopharmacology. Published by Elsevier Science Inc.
KEY WORDS: Antipsychotic; Apomorphine; Clozapine; Prepulse inhibition; Startle reflex

Animal models that can predict atypical antipsychotic action can be critically important in the development of novel therapeutic agents. Prepulse inhibition (PPI) is the normal reduction in startle amplitude that occurs when the startling stimulus is preceded by a weak prepulse (Graham 1975). PPI is disrupted in schizophrenia patients (Braff et al. 1978, 1992; Bolino et al. 1994) and in dopamine (DA)-stimulated rats, and the latter effect has been used in an animal model with face, predictive, and construct validity for the loss of PPI in

From the Department of Psychiatry, School of Medicine, University of California, San Diego, La Jolla, California.

Address correspondence to: Dr. N.R. Swerdlow, Department of Psychiatry, School of Medicine, University of California, San Diego, La Jolla, CA 92093-0804.

Received April 2, 1997; revised June 3, 1997; accepted June 11, 1997. schizophrenia patients (Swerdlow et al. 1994a). We have reported that typical antipsychotics, such as haloperidol and chlorpromazine, as well as atypical antipsychotics, such as clozapine and Seroquel, restore PPI in rats treated with the direct DA agonist apomorphine (APO) (Swerdlow et al. 1994a,b; Swerdlow and Geyer 1993). The ability of antipsychotic agents, including atypical antipsychotics, to restore PPI in APO-treated rats is strongly correlated with their clinical potency (Swerdlow et al. 1994a).

Whereas several investigators have extended our findings by demonstrating that putative atypical antipsychotics restore PPI in APO-treated rats (Cassella et al. 1994; Hoffman and Donovan 1994; Rigdon and Viik 1990; Schwarzkopf et al. 1993), others have failed to replicate our findings, casting some doubt on the general utility of this model. One recent report (Varty and Higgins 1994) failed to demonstrate the ability of subcutaneously administered clozapine to restore PPI in APO-treated Wistar rats. Because our previous report 
(Swerdlow and Geyer 1993) utilized Sprague-Dawley rats and intraperitoneal (IP) administration of clozapine, we undertook a series of experiments to examine the critical methodological differences that might account for these discrepant findings.

\section{METHODS}

Male Sprague Dawley and Wistar rats (250-272 g; Harlan Laboratories) were housed in same-strain groups of two to three and maintained on a reversed 12-h:12-h light/dark schedule (lights off at $0700 \mathrm{~h}$ ), with food and water provided ad libitum. Animals were handled individually within 3 days of arrival and daily thereafter.

Each of four startle chambers (SR-LAB, San Diego Instruments, San Diego, CA) was housed in a soundattenuated room with a $60 \mathrm{~dB}(\mathrm{~A})$ ambient noise level and consisted of a Plexiglas cylinder $8.2 \mathrm{~cm}$ in diameter resting on a $12.5 \times 25.5 \mathrm{~cm}$ Plexiglas frame within a ventilated enclosure. Acoustic noise bursts were presented via a speaker mounted $24 \mathrm{~cm}$ above the animal. A piezoelectric accelerometer mounted below the Plexiglas frame detected and transduced motion within the cylinder. The delivery of acoustic stimuli was controlled by the SR-LAB microcomputer and interface assembly which also digitized (0-4095), rectified, and recorded stabilimeter readings, with 100 1-ms readings collected beginning at startle stimulus onset. Recording gain is set and maintained constant throughout all testing at a value that prevents "clipping" of high magnitude responses. Startle magnitude was defined as the average of the 100 readings. Background noise and all acoustic stimuli were delivered through one Radio Shack Supertweeter (frequency response predominantly between $5-16 \mathrm{KHz}$ ) in each chamber. Stimulus intensities and response sensitivities were calibrated to be nearly identical in each of the four startle chambers (maximum variability $<1 \%$ of stimulus range and $<5 \%$ of response ranges). Chambers were also balanced across all experimental groups. Sound levels were measured and calibrated with a Quest Sound Level Meter, A scale (relative to $20 \mu \mathrm{N} / \mathrm{M}^{2}$ ), with the microphone placed inside the Plexiglas cylinder; response sensitivities were calibrated using an SR-LAB Startle Calibration System.

Three days before testing, each rat was placed into a startle chamber with $70 \mathrm{~dB}(\mathrm{~A})$ background noise, and 5 min later was exposed to $17118 \mathrm{~dB}(\mathrm{~A})$ 40-ms broad band bursts (PULSE) with a 15-s intertrial interval. Rats from each strain were then divided into four groups matched for mean amplitude on these trials; for each strain, these groups were assigned to receive vehicle or one of three doses of clozapine $(4,8$, or $12 \mathrm{mg} / \mathrm{kg} \mathrm{IP})$, which served as the between-subject variable. On testing days, approximately $1 \mathrm{~h}$ after arrival in the laboratory, each rat received an IP injection of vehicle or clozapine. Ten minutes later, rats received a subcutaneous (SC) injection of one of two doses of vehicle or $0.5 \mathrm{mg} / \mathrm{kg}$ $\mathrm{APO}$ and then were placed immediately into the startle chambers for a 5-min acclimation period with a 70 $\mathrm{dB}(\mathrm{A})$ background noise. The test session consisted of three trial types: (1) PULSE; (2) PULSE preceded $100 \mathrm{~ms}$ earlier by a $15 \mathrm{~dB}(\mathrm{~A})$ over background 20-ms prepulse; or (3) no stimulus (NO STIM). Sessions consisted of 63 total trials, presented in pseudorandom order, with a variable intertrial interval (range 9-21 s). One week later, this procedure was repeated, with the dose of clozapine maintained constant for each rat, and dose of APO reversed (making dose APO the within-subject variable), with APO dose order balanced across dose groups and strains.

Based on the findings of the above experiment, studies in new rats examined the effects of clozapine and a higher dose of APO $(2.0 \mathrm{mg} / \mathrm{kg})$ on PPI in Wistar rats. Sixteen rats were tested as described above, except that the pretreatment was vehicle or $8.0 \mathrm{mg} / \mathrm{kg}$ clozapine ( $n=8$ per group), and the treatment was vehicle or 2.0 $\mathrm{mg} / \mathrm{kg}$ APO. This experiment was undertaken to determine whether a dose of clozapine that significantly restored PPI in APO-treated SD rats could restore PPI in Wistar rats treated with a higher dose of APO.

Finally, the effects of clozapine on APO-disrupted PPI were examined after SC administration of clozapine. Eight SD rats were tested as described above, except that the pretreatment was vehicle or $8.0 \mathrm{mg} / \mathrm{kg}$ clozapine, administered SC ( $n=4$ per group), and the treatment was vehicle or $0.5 \mathrm{mg} / \mathrm{kg}$ APO. These rats had been tested in a previous startle session but had never received APO or clozapine, and had been drugfree for 2 weeks before participation in the present study. This experiment was undertaken to determine whether a dose of clozapine that significantly restored PPI in APO-treated SD rats after IP administration could have similar effects after administration via the SC route.

Apomorphine $\mathrm{HCl}$ (Sigma, USA) was dissolved in saline with $0.1 \mathrm{mg} / \mathrm{ml}$ ascorbic acid. Clozapine (Sandoz Pharmaceuticals, Switzerland) was dissolved in $0.1 \mathrm{~N}$ $\mathrm{HCl}$ and buffered with $\mathrm{NaOH}$ to $\mathrm{pH}$ 5.6.

All startle variables were analyzed by mixed design analyses of variance (ANOVAs). Reflex amplitude and PPI were dependent variables. PPI was defined as the percent reduction in startle amplitude in the presence of the prepulse compared to the amplitude in the absence of the prepulse [100 - $(100 \times$ amplitude on prepulse trial/amplitude on PULSE trial)]. Post hoc Tukey comparisons examined response differences between groups. Alpha was .05. 


\section{RESULTS}

In SD rats (Figure $1 A$ ) IP clozapine tended to reduce startle amplitude, as did APO, although only the latter effect reached statistical significance. ANOVA revealed no significant effect of clozapine ( $\mathrm{F}=1.22, d f 3,29, \mathrm{NS})$, a significant effect of APO ( $\mathrm{F}=4.99, d f 1,29, p<.035)$, and a nonsignificant trend toward an interaction of clozapine $\times \mathrm{APO}(\mathrm{F}=2.40$, df $3,29, p=.089)$. Whereas APO did not reduce startle amplitude in SD rats treated with clozapine vehicle, APO consistently reduced startle amplitude in SD rats treated with active doses of clozapine, an effect we have noted previously (Swerdlow et al. 1991). No significant changes in startle amplitude were noted after SC administration of clozapine in SD rats (clozapine: $\mathrm{F}=1.23, d f 1,6, \mathrm{NS}$; APO: $\mathrm{F}=4.07, d f$ 1,6 , NS; clozapine $\times$ APO interaction: $\mathrm{F}<1$; data not shown). In Wistar rats (Figure $1 B$ ), clozapine produced a highly significant, dose-dependent reduction in startle amplitude, and APO significantly increased startle amplitude. ANOVA revealed a significant effect of clozapine $(\mathrm{F}=5.49$, df 3,30, $p<.005)$, a significant effect of APO ( $\mathrm{F}=16.73$, df 1,30, $p<.0005)$, and no significant interaction of clozapine $\times \mathrm{APO}(\mathrm{F}<1)$.

In $\mathrm{SD}$ rats (Figure $2 A$ ), APO significantly reduced PPI from mean vehicle values above $80 \%$, to mean APO values below $10 \%$. This effect of APO was reversed in a dose-dependent manner by IP clozapine. ANOVA revealed a significant effect of clozapine $(\mathrm{F}=7.70, d f 3,29$, $p<.001)$, a significant effect of APO ( $\mathrm{F}=50.74, d f 1,29$, $p<.0001)$, and a significant interaction of clozapine $\times$ APO (F = 7.94, df 3,29, $p=.0005)$. Post hoc Tukey comparison revealed that PPI in APO-treated rats was increased significantly by 4,8 , and $12 \mathrm{mg} / \mathrm{kg}$ clozapine

A.

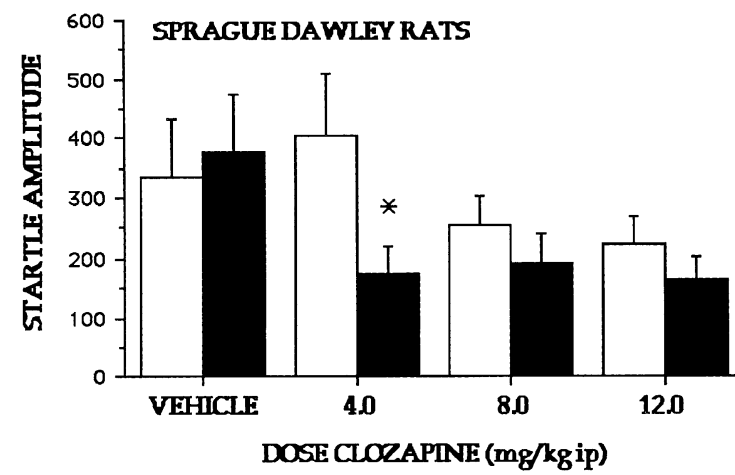

DOSE APOMORPHINE $(\mathbf{m g} / \mathrm{kgsc})$

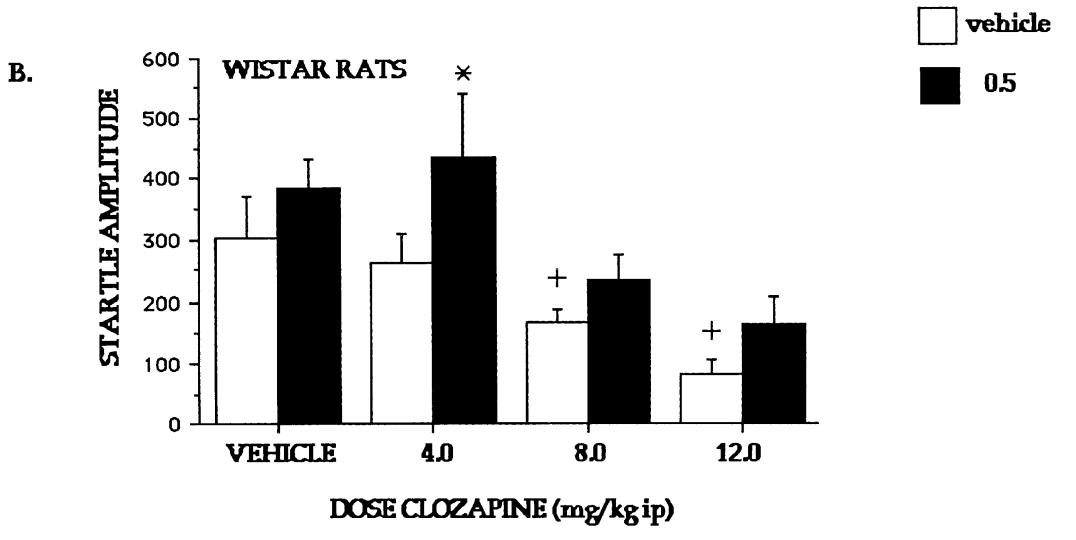

$\square$ vehicle

Figure 1. Effects of clozapine (4-12 mg/ kg IP) and APO (0.5 mg/kg SC) on baseline (pulse-alone) startle amplitude in SD rats and Wistar rats. Error bars represent SEM. (A) In SD rats, clozapine tended to reduce startle amplitude, as did APO, although only the latter effect reached statistical significance (significant main effect of APO by ANOVA). Whereas ANOVA revealed no statistically significant $\mathrm{APO} \times$ clozapine interaction, post hoc comparisons based on inspection of the data revealed that APO significantly reduced startle magnitude only in rats treated with $4.0 \mathrm{mg} / \mathrm{kg}$ clozapine $(*: \mathrm{F}=6.36, d f 1,7$, $p<.04)$. (B) In Wistar rats, clozapine produced a highly significant, dose-dependent reduction in startle amplitude $(+$ significant reduction in startle magnitude compared to rats treated with clozapine and APO vehicle, by Tukey comparison after significant main effect of clozapine by ANOVA), and APO significantly increased startle amplitude (significant main effect of APO by ANOVA). Whereas ANOVA revealed no statistically significant APO $\times$ clozapine interaction, post hoc comparisons based on inspection of the data revealed that APO significantly increased startle magnitude only in rats treated with 4.0 $\mathrm{mg} / \mathrm{kg}$ clozapine $(* \mathrm{~F}=7.98, d f 1,7, p<.03)$. 
( $p<.05$ for each dose). Route of administration did not appear to alter the ability of clozapine to restore PPI in APO-treated SD rats. ANOVA revealed a significant effect of SC clozapine $(\mathrm{F}=9.87, d f 1,6, p<.02)$, a significant effect of APO (F = 10.27, df 1,6, $p<.02)$, and a significant interaction of clozapine $\times$ APO $(\mathrm{F}=14.70, d f$ $1,6, p<.01)$. Post hoc Tukey comparison revealed that mean PPI values in APO-treated rats was increased significantly by $8 \mathrm{mg} / \mathrm{kg}$ clozapine administered via an SC route $(p<.05)$, from $26.7 \%$ to $92.6 \%$ (Figure $2 A$, inset).

To assess accurately the effects of clozapine and APO on PPI in Wistar rats, three clozapine-treated rats were eliminated from analysis whose startle amplitude levels were below 16 units (mean vehicle-group startle level = 304.1 units) (Figure 2B). In Wistar rats, APO reduced PPI from mean vehicle values of approximately $70 \%$, to mean values of approximately $45 \%$, and this effect was reversed significantly only by the highest dose of clozapine $(12 \mathrm{mg} / \mathrm{kg})$. ANOVA revealed significant effects of clozapine $(\mathrm{F}=4.50, d f 3,27, p<.015)$, and APO ( $\mathrm{F}=$ 28.01, df 1,27, $p<.0001)$, and a nonsignificant trend toward a clozapine $\times$ APO interaction $(\mathrm{F}=2.54, d f 3,27$, $p=.077)$. Inspection of the data revealed that the highest dose of clozapine $(12 \mathrm{mg} / \mathrm{kg})$ appeared to reverse the PPI-disruptive effects of APO, and a post hoc Tukey test in APO-treated rats revealed a significant $(p<.05)$ increase in PPI by the highest dose of clozapine, compared to PPI in rats treated with clozapine vehicle. Analysis of these data from Wistar rats, including the three rats with very low startle responses, revealed an identical pattern of results (statistically significant effects of clozapine and APO on PPI, significant reversal of APO effects only by the highest dose of clozapine).

Compared to findings in SD rats, APO only partially reduced PPI in Wistar rats. This APO effect appeared to be relatively less sensitive to the PPI-restorative effects of clozapine. It is possible that a more robust disruption of PPI in Wistar rats might make this measure more sensitive to the restorative effects of clozapine in this strain, by permitting more "range for improvement." To assess this possibility, 16 Wistar rats were tested as described above, after pretreatment with vehicle or 8.0 $\mathrm{mg} / \mathrm{kg}$ clozapine ( $n=8$ per group), and treatment with vehicle or $2.0 \mathrm{mg} / \mathrm{kg}$ APO.

As previously observed, APO significantly increased startle amplitude in Wistar rats. ANOVA demonstrated no significant effect of this dose of clozapine on startle amplitude ( $\mathrm{F}=2.22, d f 1,14, \mathrm{NS})$, a significant effect of $\mathrm{APO}(\mathrm{F}=14.52, d f 1,14, p<.002)$, and a near-significant interaction of $\mathrm{APO} \times$ clozapine $(\mathrm{F}=3.63, d f 1,14, p=$

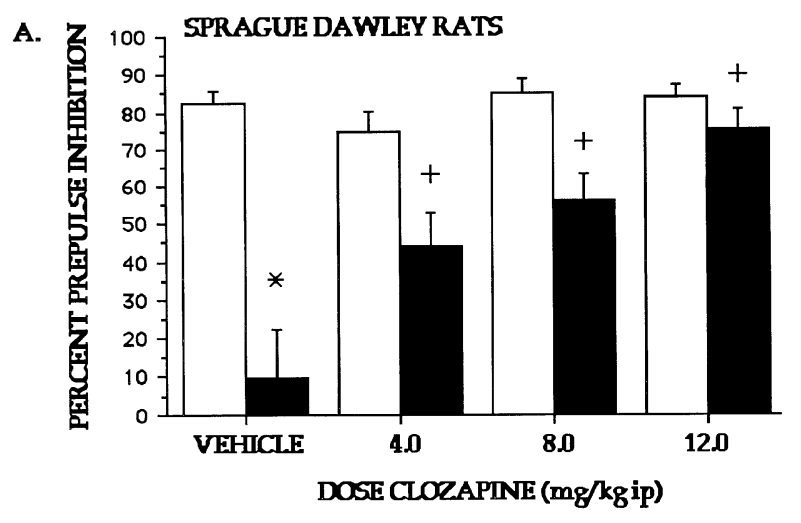

DOSE APOMORPHINE $(\mathrm{mg} / \mathrm{kg}$ sc):

vehicle

0.5
Figure 2. Effects of clozapine (4-12 mg/ $\mathrm{kg})$ and APO (0.5 mg/ $\mathrm{kg} \mathrm{SC})$ on PPI in SD rats and Wistar rats. (A) In SD rats, $\mathrm{APO}$ significantly reduced PPI $\left({ }^{*}\right)$, and this effect was reversed in a dose-dependent manner by IP clozapine (+) and by $8 \mathrm{mg} / \mathrm{kg} \mathrm{SC}$ clozapine. (B) In Wistars, APO reduced PPI to a degree substantially less than that observed in SD rats $\left({ }^{*}\right)$, and this effect was reversed significantly only by the highest dose of clozapine (12 mg/kg IP) (+).

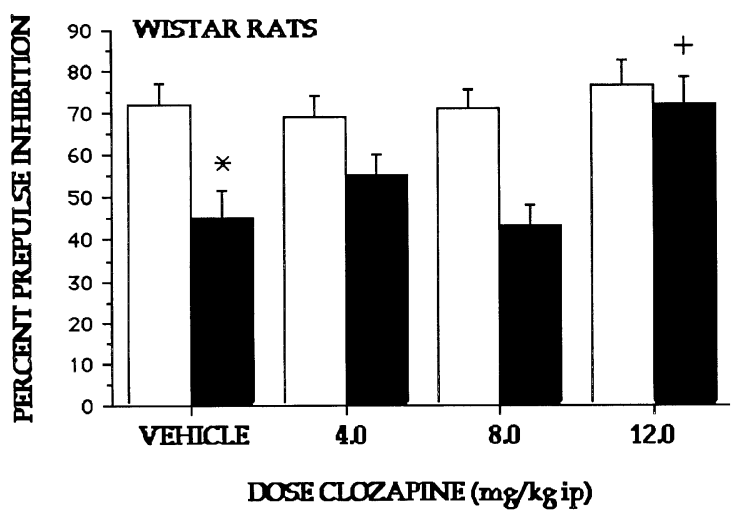


.078) (Figure 3A). The tendency for clozapine to oppose the startle-enhancing effects of APO in Wistar rats is suggested by the significant APO-induced increase in startle amplitude in rats treated with clozapine vehicle $(\mathrm{F}=14.27, d f 1,7, p<.007)$, but not in rats treated with 8 $\mathrm{mg} / \mathrm{kg}$ clozapine $(\mathrm{F}=2.12, d f 1,7, \mathrm{NS})$.

Also as previously observed, APO significantly reduced PPI in Wistar rats, and this effect was not opposed by clozapine (Figure $3 B$ ). ANOVA revealed a significant effect of APO on PPI $(\mathrm{F}=25.44, d f 1,14, p<$ $.0005)$, no significant effect of clozapine $(\mathrm{F}<1)$, and no significant APO $\times$ clozapine interaction $(\mathrm{F}=1.81, d f$ $1,14, \mathrm{NS})$. The magnitude of this effect of $2.0 \mathrm{mg} / \mathrm{kg}$ APO on PPI was comparable to that previously observed with a lower dose of APO $(0.5 \mathrm{mg} / \mathrm{kg})$. In this experiment, clozapine $(8.0 \mathrm{mg} / \mathrm{kg})$ did not oppose these APO effects: the mean APO-induced reduction in PPI was actually greater in Wistar rats pretreated with clozapine $(31.41 \%)$ than it was in Wistar rats pretreated with vehicle $(18.18 \%)$. Thus, it would be difficult to argue that the inability of clozapine to restore PPI in these $\mathrm{APO}$-treated rats reflected the restricted range of the APO effect, since the magnitude of the APO effect actually increased in clozapine-treated rats.

Whereas all statistical analyses of PPI reported above were based on percentage scores, comparisons using difference scores (startle magnitude on PULSE trial mi- nus magnitude on (prepulse + PULSE) trial) in each case yielded the expected findings: difference scores were reduced by apomorphine, to a greater degree in SD rats compared to Wistars, and clozapine reversed this effect of apomorphine, substantially or completely in SD rats (regardless of route of clozapine administration), but failed to do so in Wistars (Table 1; data reported from all rats). For example, in SD rats, apomorphine reduced difference scores from 265.23 to 33.20 in one study (IP clozapine), and from 294.96 to 85.71 in another study (SC clozapine); in these studies, clozapine elevated difference scores in apomorphine-treated rats to 114.19 (IP) and 268.58 (SC), respectively. In contrast, in Wistars, apomorphine reduced difference scores from 214.19 to 167.92 in one study $(0.5 \mathrm{mg} / \mathrm{kg} \mathrm{APO})$, whereas in another study $2.0 \mathrm{mg} / \mathrm{kg}$ of apomorphine actually increased difference scores (i.e., increased PPI) from 223.80 to 278.15; in both of these studies, clozapine actually reduced difference scores (i.e., reduced PPI) to 114.21 and 154.54, respectively.

\section{DISCUSSION}

These findings emphasize the importance of considering rat strain when attempting to replicate or extend previous findings of drug effects on PPI. Discrepant
A.
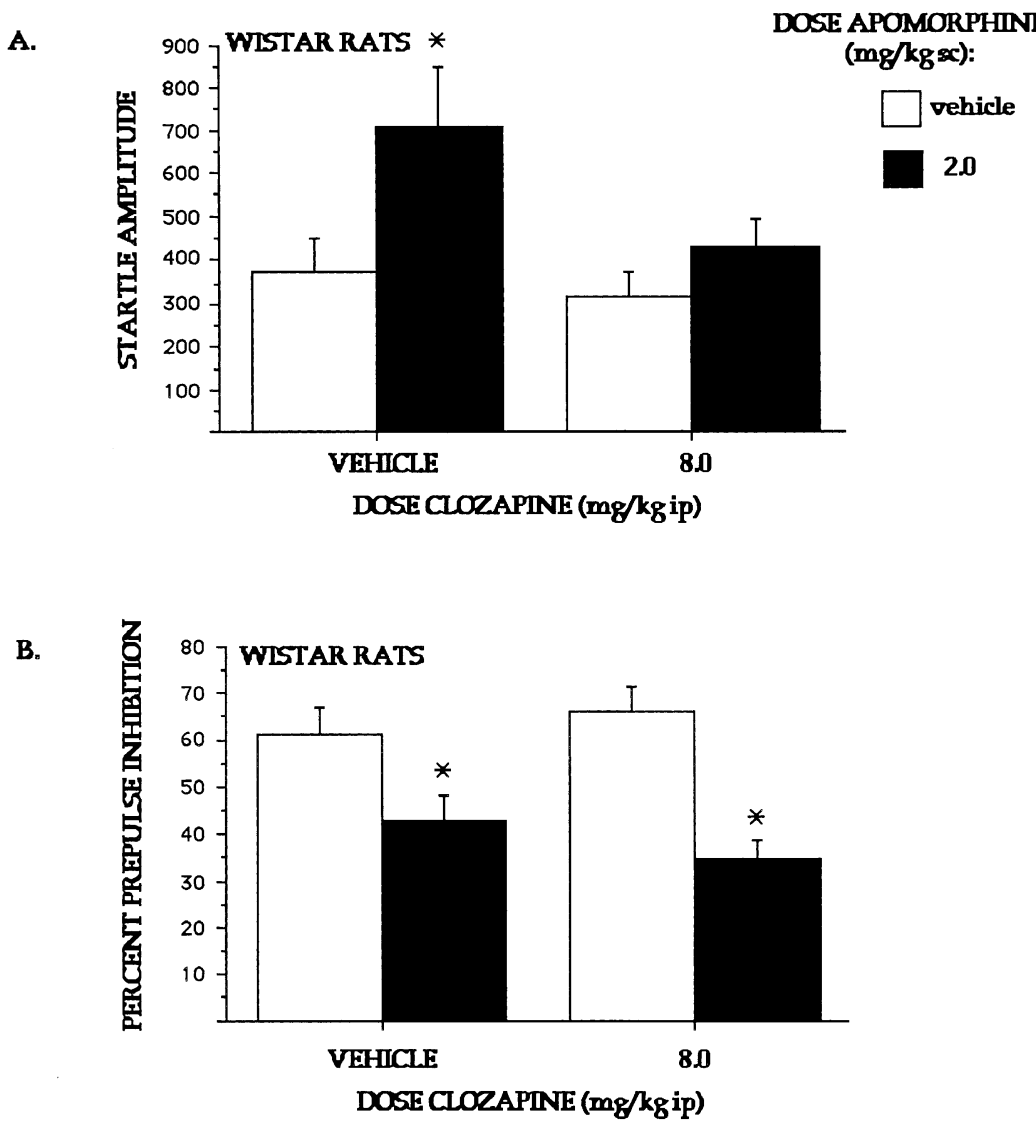

Figure 3. Effects of clozapine $(8 \mathrm{mg} / \mathrm{kg} \mathrm{IP})$ and APO (2.0 mg/kg SC) on baseline (pulsealone) startle amplitude and PPI in Wistar rats. (A) APO significantly increased startle amplitude in Wistar rats $\left(^{*}\right)$, and there was a tendency for this effect to be opposed by clozapine. (B) APO significantly reduced PPI in Wistar rats $\left(^{*}\right)$, and this effect was not opposed by clozapine. 
findings related to the ability of clozapine to restore PPI in APO-treated rats cannot be attributed solely to differences in the route of administration but appear to reflect the differences in rat strains in these studies. These results confirm that particular rat strains might be most sensitive to specific drug effects on this measure. Certainly, experimental conditions can influence the sensitivity of startle measures to several different drug effects. For example, the relative insensitivity of Wistar rats to $\mathrm{APO}$ in the present experiment may reflect the use of more intense ( $15 \mathrm{~dB}$ over background) prepulses, compared to other studies (e.g., Varty and Higgins (1994) used $5 \mathrm{~dB}$ over background prepulses). More importantly, the present findings suggest that SD rats are preferable to Wistars in studies aimed to assess "clozapinelike" effects on DA-mediated changes in PPI. It is certainly possible, however, that other "weak" drug effects on PPI in SD rats, such as the PPI-potentiating effects of nicotine, might be better studied in other rat strains.

The independence of drug effects on startle magnitude and PPI has been demonstrated in numerous studies by several different investigative groups. In the present study, clozapine and apomorphine had a variety of effects on startle magnitude, that varied across different rat strains, drug doses, and combinations. For example, in SD rats, clozapine restored PPI in apomorphine-treated rats at doses that by themselves either had no significant effect on startle magnitude (e.g., 4.0 $\mathrm{mg} / \mathrm{kg}$ ) or significantly reduced startle magnitude (e.g., $12.0 \mathrm{mg} / \mathrm{kg}$ ). In Wistar rats, doses of clozapine that significantly reduced startle magnitude (e.g., $8.0 \mathrm{mg} / \mathrm{kg}$ ), had no significant effect on PPI, and failed to restore PPI in apomorphine-treated rats. In some cases, small drug-induced changes in startle magnitude were accompanied by profound changes in PPI (e.g., in SD rats tested with $0.5 \mathrm{mg} / \mathrm{kg}$ apomorphine and $8 \mathrm{mg} / \mathrm{kg}$ clozapine SC), whereas in other cases, profound changes in startle magnitude were accompanied by minimal or no changes in PPI (e.g., in Wistar rats treated with 2.0 $\mathrm{mg} / \mathrm{kg}$ apomorphine and $8 \mathrm{mg} / \mathrm{kg}$ clozapine IP). The fact that drug effects on PPI were evident whether they were calculated by percentage scores (Figures 2-3) or difference scores (Table 1) also suggests that these effects are not simply an artifact of drug-induced changes in startle magnitude.

The biological basis for these strain differences in clozapine sensitivity might be relevant to our understanding of the neural mechanisms of clozapine effects, which remain poorly understood, despite intensive study. Furthermore, strain differences in the PPI-disruptive effects of particular drugs (Rigdon 1990; present data) might be quite relevant to "strain-related" differences in the manifestations of human disorders of information processing, including schizophrenia. The molecular basis for such strain differences in rats might be studied effectively via pharmacogenetic strategies, sim-

Table 1. Mean Startle Amplitudes and Difference Scores in Sprague Dawley (SD) and Wistar Rats

\begin{tabular}{lccccc}
\hline Strain & $\begin{array}{c}\text { Clozapine } \\
(\mathbf{m g} / \mathbf{k g})\end{array}$ & $\begin{array}{c}\text { Apomorphine } \\
(\mathbf{m g} / \mathbf{k g})\end{array}$ & $\begin{array}{c}\text { Pulse } \\
\text { Alone }\end{array}$ & Prepulse + Pulse & $\begin{array}{c}\text { Difference } \\
\text { Score }\end{array}$ \\
\hline SD & veh & veh & 335.10 & 69.86 & 265.23 \\
SD & 4 & veh & 405.59 & 107.95 & 297.64 \\
SD & 8 & veh & 254.13 & 48.74 & 205.39 \\
SD & 12 & veh & 222.45 & 36.62 & 185.82 \\
SD & veh & 0.5 & 375.45 & 342.25 & 33.20 \\
SD & 4 & 0.5 & 174.99 & 110.55 & 64.43 \\
SD & 8 & 0.5 & 190.95 & 86.77 & 104.18 \\
SD & 12 & 0.5 & 164.79 & 50.61 & 114.19 \\
Wistar & veh & veh & 304.13 & 89.93 & 214.19 \\
Wistar & 4 & veh & 262.79 & 89.67 & 173.13 \\
Wistar & 8 & veh & 169.86 & 52.80 & 117.06 \\
Wistar & 12 & veh & 80.80 & 17.07 & 63.72 \\
Wistar & veh & 0.5 & 383.10 & 215.18 & 167.92 \\
Wistar & 4 & 0.5 & 435.68 & 214.43 & 221.24 \\
Wistar & 8 & 0.5 & 232.96 & 140.89 & 92.07 \\
Wistar & 12 & 0.5 & 166.18 & 51.96 & 114.21 \\
SD & veh (SC) & veh & 347.05 & 52.08 & 294.96 \\
SD & $8(S C)$ & veh & 239.50 & 43.30 & 196.20 \\
SD & veh (SC) & 0.5 & 494.92 & 409.20 & 85.71 \\
SD & $8(S C)$ & 0.5 & 308.06 & 39.48 & 268.58 \\
Wistar & veh & veh & 374.35 & 150.54 & 223.80 \\
Wistar & 8 & veh & 317.69 & 118.31 & 199.38 \\
Wistar & veh & 2.0 & 711.18 & 433.03 & 278.15 \\
Wistar & 8 & 2.0 & 429.97 & 275.43 & 154.54 \\
\hline & & & & &
\end{tabular}


ilar to those applied toward understanding the genetic control of the DAergic regulation of PPI (Ellenbroek et al. 1995).

\section{ACKNOWLEDGMENTS}

This work was supported by NIMH grants MH 48381 and MH 01436 (NRS), MH 42228 (MAG and NRS), MH 52885 (MAG and NRS), and MH 01223 (MAG). We gratefully acknowledge the expert assistance of Navid Taaid, Pamela Auerbach, and Marianne Dalmus.

\section{REFERENCES}

Bolino F, Di Michele V, Di Cicco L, Manna V, Daneluzzo E, Cassachia M (1994): Sensorimotor gating and habituation evoked by electrocutaneous stimulation in schizophrenia. Biol Psychiatry 36:670-679

Braff D, Stone C, Callaway E, Geyer M, Glick I, Bali L (1978): Prestimulus effects on human startle reflex in normals and schizophrenics. Psychophysiology 15:339-343

Braff DL, Grillon C, Geyer M (1992): Gating and habituation of the startle reflex in schizophrenic patients. Arch Gen Psychiatry 49:206-215

Cassella J, Hoffman D, Rajachandran L, Donovan H, Bankoski C, Lang S, Johnson A, Thurkauf A, Hutchison A (1994): The behavioral profile of NGD 94-1, a potent and selective dopamine D4 receptor antagonist. Proceedings Amer College Neuropsychopharmacol, p. 228

Ellenbroek BA, Geyer MA, Cools MA (1995): The behavior of APO-SUS rats in animal models with construct validity for schizophrenia. J Neurosci 15:7604-7611
Graham F (1975): The more or less startling effects of weak prestimuli. Psychophysiology 12:238-248

Hoffman DC, Donovan H (1994): $\mathrm{D}_{1}$ and $\mathrm{D}_{2}$ dopamine receptor antagonists reverse prepulse inhibition deficits in an animal model of schizophrenia. Psychopharmacology 115:447-453

Rigdon G (1990): Differential effects of apomorphine on prepulse inhibition of acoustic startle reflex in two rat strains. Psychopharmacology 102:419-421

Rigdon GC, Viik K (1991): Prepulse inhibition as a screening test for potential antipsychotics. Drug Dev Res 23:91-99

Schwarzkopf SB, Bruno JP, Mitra T (1993): Effects of haloperidol and SCH 23390 on acoustic startle and prepulse inhibition under basal and stimulated conditions. Prog Neuropsychopharmacol Biol Psychiatry 17:1023-1036

Swerdlow NR, Keith VA, Braff DL, Geyer MA (1991): The effects of spiperone, raclopride, SCH 23390 and clozapine on apomorphine-inhibition of sensorimotor gating of the startle response in the rat. J Pharmacol Exp Ther 256:530-536

Swerdlow NR, Geyer MA (1993): Clozapine and haloperidol in an animal model of sensorimotor gating deficits in schizophrenia. Pharmacol Biochem Behav 44:741-744

Swerdlow NR, Braff DL, Taaid N, Geyer MA (1994a): Assessing the validity of an animal model of sensorimotor gating deficits in schizophrenic patients. Arch Gen Psychiatry 51:139-154

Swerdlow NR, Zisook D, Taaid N (1994b): Seroquel (ICI $204,636)$ restores prepulse inhibition of acoustic startle in apomorphine-treated rats: Similarities to clozapine. Psychopharmacology 114:675-678

Varty GB, Higgins GA (1994): Differences between three rat strains in sensitivity to prepulse inhibition of acoustic startle response: influence of apomorphine and phencyclidine pretreatment. J Psychopharmacology 8:148-156 